EPJ manuscript No.

(will be inserted by the editor)

\title{
New experimental results in proton radioactivity
}

\author{
K. P. Rykaczewski ${ }^{1,2}$ \\ 1 Physics Division, Oak Ridge National Laboratory, Oak Ridge, TN 37831 \\ 2 Institute of Experimental Physics, Warsaw University, Pl-00681 Warsaw, Poland
}

Received: date / Revised version: date

\begin{abstract}
A review of experimental data obtained recently on proton-radioactive nuclei is presented. The highlights include the observation of fine structure in proton emission, for the decays of ${ }^{131} \mathrm{Eu},{ }^{145} \mathrm{Tm}$ and ${ }^{146} \mathrm{Tm}$, and the studies of the excited states in proton-emitting nuclei. The observation limits are extended to few nanobarns cross sections $\left({ }^{140} \mathrm{Ho},{ }^{164} \mathrm{Ir}\right.$ and $\left.{ }^{130} \mathrm{Eu}\right)$ and few microsecond half-lives (e.g. $\left.{ }^{145} \mathrm{Tm}\right)$. Measured decay properties for thirty nine proton-emitting ground and isomeric states contributed to the understanding of nuclear masses and evolution of single-particle states at and beyond the proton drip-line. Experimental results have stimulated new theoretical approaches to proton emission and the structure of unbound narrow resonance states.
\end{abstract}

PACS. $23.50 .+z$ Decay by proton emission -21.10 -k Properties of nuclei

\section{Introduction - Proton Radioactivity}

Proton radioactivity studies provide a unique insight into the structure of nuclei beyond the drip-line limit. The evolution of the single-particle structure, nuclear shapes and masses can be deduced from measured properties of proton emission. Recent progress in the experiment and theory made possible the analysis of the composition of the wave function of these narrow unbound resonance states.

The first proton-radioactive state, a metastable level in ${ }^{53} \mathrm{Co}$ was discovered over thirty years ago [1]. Till now, it remains the only proton radioactivity observed below $\mathrm{Z}=50$. All other thirty eight experimentally known proton emitters have atomic numbers between $\mathrm{Z}=51$ and $\mathrm{Z}=83$. An experimental observation window for proton radioactivity is relatively wide for the neutron-deficient nuclei in the region from $\mathrm{Sn}$ to $\mathrm{Pb}$ elements. It is a joint effect of the mass surface and the presence of proton orbitals with a wide range of angular momentum (from $l=0$ to $l=5)$ near Fermi level. Also, these nuclei beyond the proton drip-line can be reached and studied with fusionevaporation reactions. For several combinations of stable projectile and target, the proton drip-line is crossed far enough to detect proton emission already for $p 2 n$ fusionevaporation channel. A typical cross section is around few tens of microbarns. Even with a small proton branching ratio caused by the competition of $\alpha$ and/or $\beta$-decay, the proton lines can be observed. Several of these emitters have halflives in the milliseconds range. Even with a delay in the ion source, the proton activity could be detected with the on-line mass separator technique [2]. However, the proton decay width increases rapidly with the departure from the beta stability line resulting in microsecond half-lives. Very fast separation of reaction products is necessary. Most of the known proton emitters were discovered using fusion-evaporation reactions studied by the means of recoil separation and segmented Sidetectors : at SHIP (GSI Darmstadt), at DRS (Daresbury), at FMA (Argonne), at RMS (Oak Ridge), and most recently at RMS (Legnaro) and RITU (Jyväskylä). The past few years have seen an explosion of both experimental and theoretical work on the topic of proton emission. Within last three years new experimental data on twenty four proton-radioactive nuclei were announced. The observation limits were extended to very low cross sections and very short halflives. The $p 5 n$ fusion-evaporation reaction channel characterized by a cross section even below 10 nanobarns was successfully used to identify the activities of ${ }^{140} \mathrm{Ho}$ [3], ${ }^{164} \mathrm{Ir}[4,5]$ and ${ }^{130} \mathrm{Eu}$ [5]. The odd-odd emitter ${ }^{164} \mathrm{Ir}$ is the fourth isotope of iridium where proton emission was observed. Seven proton radioactivities with halflives below 50 microseconds are known up to date. The first one, the $18 \mu$ s activity of ${ }^{113} \mathrm{Cs}$ was identified about 20 years ago in Munich [6], and later restudied at GSI, Daresbury, Oak Ridge and Argonne. The next six emitters ${ }^{141 m} \mathrm{Ho},{ }^{145} \mathrm{Tm},{ }^{150 m} \mathrm{Lu},{ }^{151 m} \mathrm{Lu},{ }^{155} \mathrm{Ta}$ and ${ }^{171} \mathrm{Au}$ were observed within last years. The digital processing of detector signals [7] allows us to start a search for the activities with submicrosecond halflives, as demonstrated by the recent study on the decay of $3-\mu$ s activity of ${ }^{145} \mathrm{Tm}$ [8-10].

The experimental investigations of proton radioactivity are not limited to the decay spectroscopy. Excited levels above proton-emitting states are deduced from prompt $\gamma$-radiation measured at the target area. The $\gamma$-cascades feeding the proton-radioactive level are selected by tagging 
K. P. Rykaczewski: New experimental results in proton radioactivity

on the proton emission signals recorded at the focal plane of recoil separators (Recoil Decay Tagging method). Powerful combinations of detectors, Gammasphere coupled to the FMA (Argonne), and CLARION coupled to the RMS (Oak Ridge) allowed us to obtain first information on the excited levels in ${ }^{109} \mathrm{I}[11],{ }^{113} \mathrm{Cs}$ [12], ${ }^{131} \mathrm{Eu}[13],{ }^{141 m, g s} \mathrm{Ho}$ [14], ${ }^{151} \mathrm{Lu}[15]$ and ${ }^{167} \mathrm{Ir}[16]$.

Proton emission was commonly used to deduce the properties of proton orbitals. Recent observation of a fine structure in proton decay of the odd-odd isotope ${ }^{146} \mathrm{Tm}$ represents the first study of neutron states in exotic nuclei populated by proton emission.

Selected results on proton radioactivity achieved within last few years are discussed below.

\section{Fine structure in proton emission}

The decay width for proton emission depends very strongly on the available energy. Spherical even-even nuclei have their first excited state at least at few hundred $\mathrm{keV}$ above the ground state. Therefore, proton transitions from (odd$\mathrm{Z}$, even-N) nuclei to the excited levels in the daughter nucleus were not observed during many years of experiments on proton radioactivities. In recent years the proton drip-line has been crossed in the region of well deformed isotopes. The experiments at the FMA (Argonne) and RMS (Oak Ridge) reported the identification of ${ }^{131} \mathrm{Eu}[17]$, ${ }^{140} \mathrm{Ho}$ and ${ }^{141 g s, m} \mathrm{Ho}[17,3]$. Recently, ${ }^{117} \mathrm{La}$ was identified at the RMS, Legnaro [18] and investigated at the FMA, Argonne [19].

The energies of excited first $2^{+}$state in ${ }^{130} \mathrm{Sm}$ and ${ }^{140}$ Dy were expected at about $120 \pm 20 \mathrm{keV}$ and $160 \pm 20$ $\mathrm{keV}[20,21]$. The pioneering experiment on ${ }^{131} \mathrm{Eu}$ radioactivity at the FMA resulted in the detection of two proton transitions of $932 \mathrm{keV}$ and $811 \mathrm{keV}$ [22]. Based on similar halflives, these two proton lines were assigned to the decay of 18 millisecond activity of ${ }^{131} \mathrm{Eu}$ populating the $0^{+}$and $2^{+}$states in ${ }^{130} \mathrm{Sm}$. The measured branching ratio to the $2^{+}$state, of about $24 \pm 5 \%$, together with the decay energies and halflife pointed to the $3 / 2^{+}[411]$ groundstate of ${ }^{131} \mathrm{Eu}$. The low $2^{+}$energy of $121 \mathrm{keV}$ in ${ }^{130} \mathrm{Sm}$ confirmed the large deformation $\beta_{2} \approx 0.34$ expected for nuclei in this region. The most advanced theoretical analysis of this $\mathrm{I}^{\pi}=3 / 2^{+}$state was done within the non-adiabatic coupled-channel method $[23,24]$. It show that the main components of this wave function are the $\mathrm{d}_{5 / 2}(67 \%), \mathrm{g}_{7 / 2}$ $(17 \%)$ and $g_{9 / 2}(10 \%)$ spherical proton orbitals, see Fig.1.

However, the decay width for the ground-state proton transition in the decay of ${ }^{131} \mathrm{Eu}$ is dominated by a small admixture of the $d_{3 / 2}$ orbital, of about $1 \%$ of the total wave function. The width of the proton decay to the excited $2^{+}$state is a result of mostly $d_{5 / 2}$ component, with small part arising from $\mathrm{d}_{3 / 2}, \mathrm{~s}_{1 / 2}$ and $\mathrm{g}_{7 / 2}$ orbitals, see Fig.2.

This rather complex picture could be at least partially corroborated by the experimental information on the excited states in ${ }^{131} \mathrm{Eu}$. A Recoil Decay Tagging experiment on ${ }^{131} \mathrm{Eu}$ radioactivity was performed with the Gammasphere and FMA [13]. Besides investigating the structure

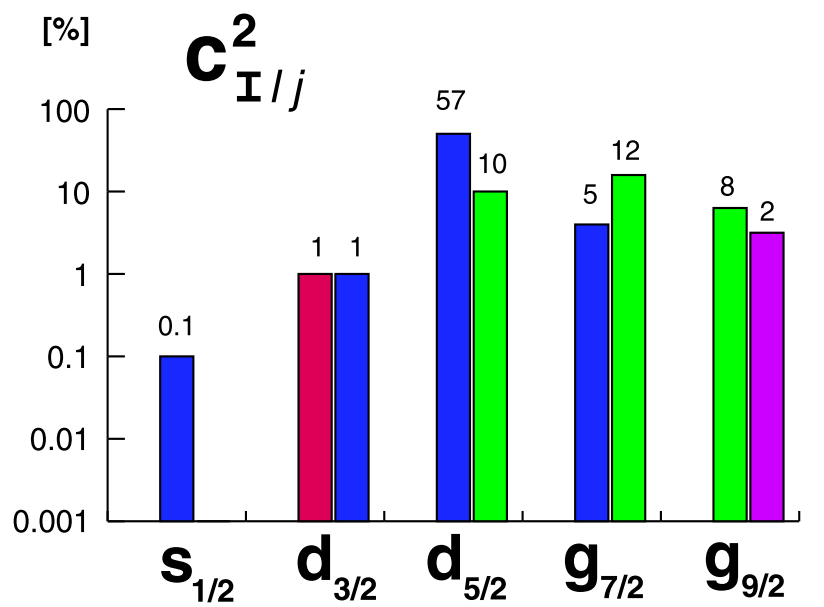

Fig. 1. Structure of the $3 / 2^{+}[411]{ }^{131} \mathrm{Eu}$ ground-state as composed of the spherical $l j$ orbitals $\mathrm{s}_{1 / 2}, \mathrm{~d}_{3 / 2}, \mathrm{~d}_{5 / 2}, \mathrm{~g}_{7 / 2}$ and $\mathrm{g}_{9 / 2}$. The fractions of the wave function coupled to the final $\mathrm{I}=0^{+}$ (red), $2^{+}$(blue), $4^{+}$(green) and $6^{+}$(violet) states in ${ }^{130} \mathrm{Sm}$ via proton emission are indicated.

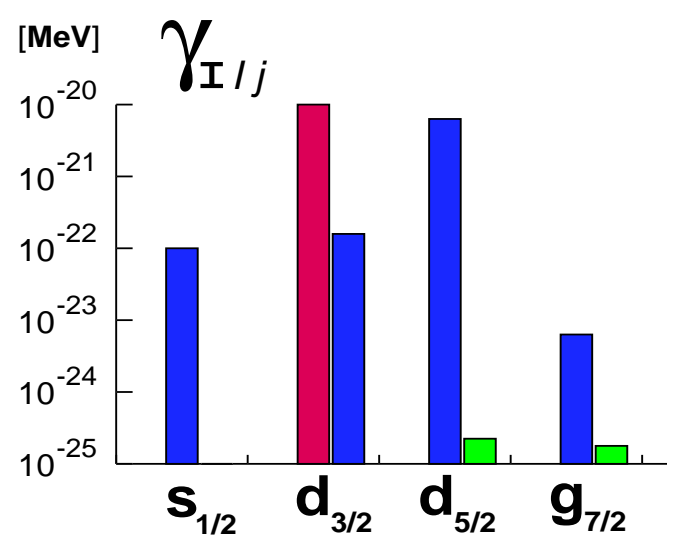

Fig. 2. Main components of the decay width (in $\mathrm{MeV}$ ) for the proton transitions from the ${ }^{131} \mathrm{Eu}$ ground-state to the $\mathrm{I}=0^{+}$ ground state (red) and to the excited $\mathrm{I}=2^{+}$(blue) and $\mathrm{I}=4^{+}$ (green) states of ${ }^{130} \mathrm{Sm}$.

of excited states, it was an attempt to confirm independently the origin of both proton lines from the same state. Since the cross section for production of ${ }^{131} \mathrm{Eu}$ is very low, about $70 \mathrm{nb}$, and there are several bands fractioning the total gamma intensity - the result is not conclusive yet.

The search for fine structure in the decay of both pradioactive states in ${ }^{141} \mathrm{Ho}$ resulted in the upper limit for the branching ratio to the $2^{+}$state in ${ }^{140} \mathrm{Dy}$, of about $1 \%$ for both emitters [14]. It is likely that the energy of this $2^{+}$state is above the expected $160 \pm 20 \mathrm{keV}[20,21]$. This conclusion follows the theoretical analysis of the observed proton decay rates, and is suggested by results of the RDT study of ${ }^{141} \mathrm{Ho}$ [14]. The $\beta_{2} \approx 0.25$ with significant hexadecapole deformation and even triaxial shape for the ground-state was deduced for this nucleus from the observed excited levels pattern [14]. Such $\beta_{2}$ value would imply $\mathrm{E}\left(2^{+}\right) \approx 190 \mathrm{keV}[25]$. Experiments to determine this 


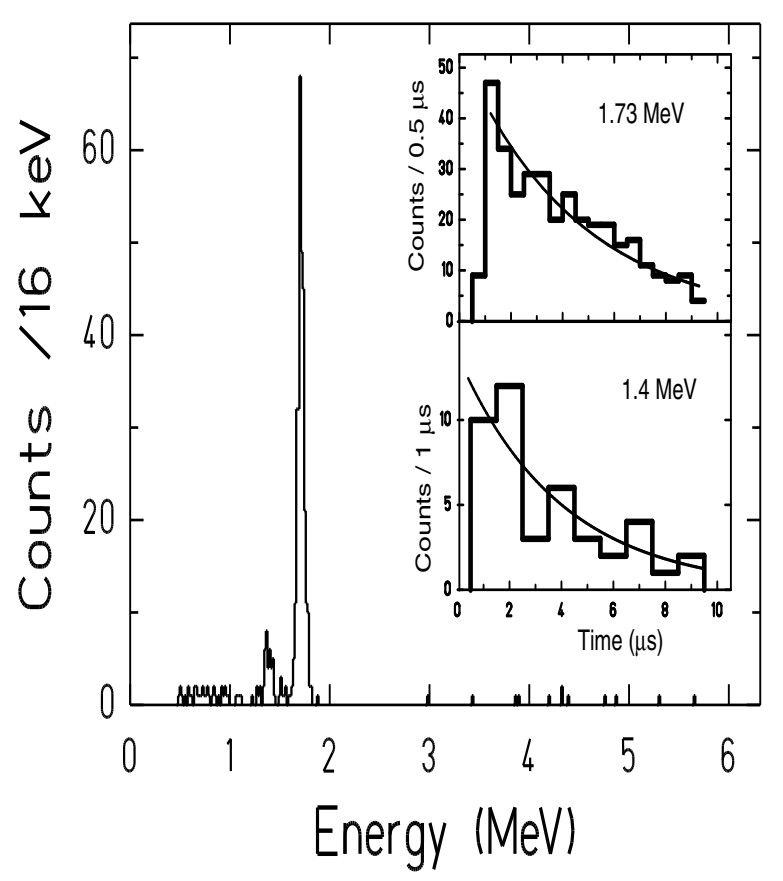

Fig. 3. An energy spectrum and decay patterns of proton lines originating from ${ }^{145} \mathrm{Tm}$ radioactivity.

energy value via the decay spectroscopy of expected shortlived $\mathrm{I}^{\pi}=8^{-}$metastable state in ${ }^{140}$ Dy were attempted at Jyväskylä [26,27], and are proposed at Oak Ridge and Argonne.

Fine structure in proton emission was also recently detected for the decay of the proton emitter with the shortest halflife known to date, the 3-microsecond activity of ${ }^{145} \mathrm{Tm}$ [8-10]. Thanks to the new data acqusition system based on the digital signal processing [7], the detection rate for ${ }^{145} \mathrm{Tm}$ decay events was increased by about an order of magnitude. In addition to the known proton transition at $1.73 \mathrm{MeV}$ [8], a new line at $1.4 \mathrm{MeV}$ with similar halflife was observed, see Fig. 3 .

The proton line at $1.4 \mathrm{MeV}$ is interpreted as the transition to the $2^{+}$state at $0.33 \mathrm{MeV}$ in the daughter nucleus ${ }^{144} \mathrm{Er}$. This energy fits the expectations from simple $\mathrm{N}_{p} \mathrm{~N}_{h}$ systematics $[20,21]$, i.e. it is close to $\mathrm{E}\left(2^{+}\right)=343 \mathrm{keV}$ known for the " $\mathrm{N}=82$ mirror" nucleus ${ }^{156} \mathrm{Er}$. It leads to an estimate of $\beta_{2} \approx 0.18$ [25] for ${ }^{144} \mathrm{Er}$. The wave function of such transitional system can be described using particle-vibration coupling [28-31]. The main component is, as expected, the $\pi \mathrm{h}_{11 / 2}$ coupled to $0^{+}$state of ${ }^{144} \mathrm{Er}$. It is enough to have 3 to $5 \%$ admixture of the $\pi \mathrm{f}_{7 / 2} \otimes 2^{+}$to explain observed branching ratio of about $9 \%$. The second largest component of the wave function, the $\pi \mathrm{h}_{11 / 2} \otimes 2^{+}$, doesn't contribute significantly to the decay width to the $2^{+}$state.

Studies of proton emission from ground- and isomericstates in the rare-earth region were used to deduce the properties of proton orbitals beyond the drip-line. How- ever, in this region of nuclei the same orbitals, the $\mathrm{h}_{11 / 2}$, $\mathrm{d}_{3 / 2}$ and $\mathrm{s}_{1 / 2}$, are occupied by protons and by neutrons. These $\nu$-orbitals are close to the Fermi surface and create the neutron states at low excitation energies in even-Z, odd-N nuclei. Such low energy states might have observable population by proton transitions following the decays of odd-N odd-Z emitters. This idea has triggered the experiments on odd-odd proton emitters ${ }^{146} \mathrm{Tm}$ and ${ }^{150} \mathrm{Lu}$ at Oak Ridge. A new short-lived isomeric state ${ }^{150 m} \mathrm{Lu}$ was discovered $[32,33]$, but no evidence for the fine structure was obtained for both emitters, the ${ }^{150 g s} \mathrm{Lu}$ and ${ }^{150 m} \mathrm{Lu}$. However, the experiment on ${ }^{146} \mathrm{Tm}$ revealed the presence of three new proton transitions at $0.89 \mathrm{MeV}, 0.94 \mathrm{MeV}$ and $1.02 \mathrm{MeV}$, in addition to the two known lines at 1.12 $\mathrm{MeV}$ and $1.19 \mathrm{MeV}$, see [34,9,35]. The decay patterns suggested the assignment of the $0.89 \mathrm{MeV}$ line to the decay of a $\mathrm{T}_{1 / 2}=200 \mathrm{~ms}$ state, and the $0.94 \mathrm{MeV}$ transition to the $85 \mathrm{~ms}$ level. Following known structure of heavier oddodd thulium isotopes, these levels are interpreted as the $\left(10^{+}\right)$isomer and $\left(6^{-}\right)$ground-state. Within the spherical picture, the wave function of ${ }^{146 \mathrm{~g} s} \mathrm{Tm}$ is dominated by the $\pi \mathrm{h}_{11 / 2} \otimes \nu \mathrm{s}_{1 / 2}$ configuration. The main proton transition at $1.19 \mathrm{MeV}$ populates the $\nu_{\mathrm{s}_{1} / 2}$ ground-state of even-Z,odd-N ${ }^{145}$ Er. A small admixture $(\approx 1 \%)$ of mirror configuration, the $\nu \mathrm{h}_{11 / 2} \otimes \pi \mathrm{s}_{1 / 2}$, is enough to observe the lower energy $l=0$ transition to the excited $\nu \mathrm{h}_{11 / 2}$ state at $250 \mathrm{keV}$ in ${ }^{145} \mathrm{Er}$. The $\left(10^{+}\right)$state resulting from the coupling of $\mathrm{h}_{11 / 2}$ proton and $\mathrm{h}_{11 / 2}$ neutron decays to the same $250 \mathrm{keV} \nu \mathrm{h}_{11 / 2}$ state via the $1.12 \mathrm{MeV}$ transition. Weak 0.89 line is likely the $l=3$ proton emission to the excited state at $0.48 \mathrm{MeV}$ originating from small $\pi \mathrm{f}_{7 / 2} \otimes 2^{+}$ component "replacing" the $\pi \mathrm{h}_{11 / 2}$ part in the $\mathrm{I}^{\pi}=\left(10^{+}\right)$ wave function, compare the decay of neighboring ${ }^{145} \mathrm{Tm}$. The $1.02 \mathrm{MeV}$ transition cannot be placed unambiguosly in the decay scheme yet.

\section{Proton emission from near-spherical $\mathbf{I}^{\pi}=3 / 2^{+}$states}

Proton emission rates for spherical nuclei are well analysed theoretically [36]. In an advanced approach the tunneling probability is calculated within a two-potential approach with an account for observed proton energy. The occupation of each respective proton orbital is an important factor influencing decay rate [36]. The measured halflife values are well reproduced for $l=5$ and $l=0$ proton emissions. However, for the $l=2$ emission the calculated decay width was about two times too large, see Fig. 4.

This discreapancy was first discussed by P.Semmes [28, 29] for the decay of ${ }^{151 m} \mathrm{Lu}$ [37]. The wave function of observed proton-emitting $\mathrm{I}^{\pi}=3 / 2^{+}$states is not a pure $\pi \mathrm{d}_{3 / 2}$ coupled to the $0^{+}$even-even core. There are substantial components with $\mathrm{s}_{1 / 2}$ and $\mathrm{d}_{3 / 2}$ protons coupled to the $2^{+}$ configuration of the respective daughter nucleus. These "contaminations" are responsible for reducing the proton transition rate from the complex $\mathrm{I}^{\pi}=3 / 2^{+}$level to the final $0^{+}$state. The presence of such components might be manifested by the proton transition to the excited $2^{+}$state. 


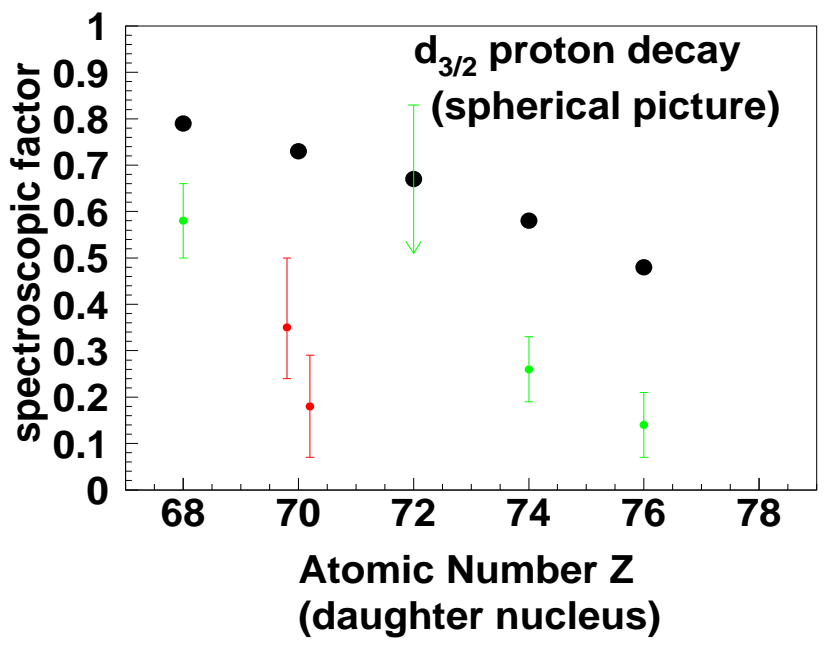

Fig. 4. Spectoscopic factors for the $l=2$ proton emission analysed asuming spherical $\pi \mathrm{d}_{3 / 2} \otimes 0^{+}$parent state configuration. Red symbols denote recently observed decays of ${ }^{150 m} \mathrm{Lu}$ and ${ }^{151 m} \mathrm{Lu}$. Black dots indicate the calculated vacancies $u^{2}$ for respective $\pi \mathrm{d}_{3 / 2}$ states.

The energy of such first excited state for near-spherical rare-earth nuclei is above $\approx 500 \mathrm{keV}$. The decay width to the $2^{+}$state is well below present observation limits. The account for more complex configuration was made also for spherical and near-spherical $\pi \mathrm{h}_{11 / 2}$ and $\pi \mathrm{s}_{1 / 2}$ states $[28,29]$. It did not spoil the good agreement between experimental and calculated spectroscopic factors. Very recently, more detailed descriptions of proton emission analysed within the particle-vibration coupling model become available $[30,31]$.

\section{Summary and outlook}

Many new results on proton radioactive nuclei were presented within last few years. Observation limits were pushed with respect to the production cross section, halflife and branching ratio. Experimentally, we are getting ready to profit from production methods new for proton radioactiviy studies like the fragmentation of heavy ions [7] and use of postaccelerated radioactive beams [38]. The challenge for the coming years include the identification of new emitters and further studies on the fine structure in proton emission. These studies are important for the verification and extension of the present description of exotic nuclei. In particular, for the region at and below doublymagic ${ }^{100} \mathrm{Sn}$, experiments on proton emission should provide also an input for the analysis of rp-process nucleosynthesis. Studies of odd-odd emitters might allow us to deduce more information on exotic neutron orbitals populated in the proton emission. It remains to be seen how unique the decay properties of odd-odd ${ }^{146} \mathrm{Tm}$ are. Future studies might involve oriented nuclei [39] and the measurements of angular distributions [40,41]. It should define the orbital angular momentum carried by emitted proton verifying the interpretation based on the decay rates.

ORNL is managed by UT-Batelle,LLC, for the U.S. Department of Energy under contract DE-AC-00OR22725.

\section{References}

1. K.P. Jackson et al.: Phys. Lett. 33B (1970) 281

2. O. Klepper et al: Z. Phys. A305, (1982) 125

3. K. Rykaczewski et al.: Phys. Rev. C60 (1999) 011301

4. H. Kettunen et al.: Acta Phys. Pol. B32 (2001) 989.

5. C.N. Davids et al.: this proceedings.

6. T. Faestermann et al: Phys. Lett. B137 (1984) 23

7. R.K. Grzywacz et al.: this proceedings.

8. J.C. Batchelder et al.: Phys. Rev. C57 (1998) R1042

9. K.P. Rykaczewski et al.: Nucl. Phys. A682 (2001) 270c

10. M. Karny et al.: to be published

11. C.-H. Yu et al.: Phys. Rev. C59 (1999) R1834

12. C.J. Gross et al.: in Proc. of Int. Conf. on Exotic Nuclei and Atomic Masses ENAM 98, Bellaire, Michigan, June 1998, eds. B.M. Sherrill, D.J. Morrisey and C.N. Davids; AIP 455, Woodbury, New York (1998) 444

13. D. Seweryniak et al.: Nucl. Phys. A682 (2001) 247c

14. D. Seweryniak et al.: Phys. Rev. Lett. 86 (2001) 1458

15. C.-H. Yu et al.: Phys. Rev. C58 (1998) R3042

16. M.P. Carpenter : Acta Phys. Pol. B30 (1999) 581

17. C.N. Davids et al.: Phys. Rev. Lett. 80 (1998) 1849

18. F. Soramel et al: Phys. Rev. C63 (2001) 031304(R)

19. H. Mahmud et al.: Phys. Rev. C64 (2001) 031303(R)

20. R.F. Casten: Phys. Rev. C33 (1986) 1819

21. R.F. Casten and N.V. Zamfir: J. Phys. G22 (1996) 1521

22. A.A. Sonzogni et al.: Phys. Rev. Lett. 83 (1999) 1116

23. A.T. Kruppa et al: Phys. Rev. Lett. 86 (2000) 4549

24. B. Barmore et al.: Phys. Rev. C62 (2000) 054315

25. L. Grodzins: Phys. Lett. 2 (1962) 88

26. D. Cullen et al:: Phys. Rev. C58 (1998) 846

27. C. Scholey et al.: Phys. Rev. C63 (2001) 034321.

28. P.B. Semmes: in Proc. of Int. Symp. on Proton-Emitting Nuclei, Oak Ridge, TN, 1999, ed. J.C. Batchelder, AIP 518 (2000) 125

29. P.B. Semmes: Nucl. Phys. A682 (2001) 239c

30. C.N. Davids and H. Esbensen: Phys. Rev. C (2001) in press.

31. K. Hagino: Phys. Rev. C (2001) in press.

32. T.N. Ginter et al.: Phys. Rev. C61, 014308 (1999)

33. K.P. Rykaczewski et al.: Acta Phys. Pol. B32 (2001) 971.

34. T.N. Ginter et al.: in Proc. of Int. Symp. on ProtonEmitting Nuclei, Oak Ridge, TN, 1999, ed. J.C. Batchelder, AIP 518 (2000) 83

35. J.C. Batchelder et al: this proceedings.

36. S. Aberg et al.: Phys. Rev. C56 (1997) 1762 and Phys. Rev. C58 (1998) 3011

37. C. R. Bingham et al.: Phys. Rev. C59 (1999) R2984

38. J. Gomez del Campo et al: Phys. Rev. Lett. 86 (2001) 43

39. D. Rudolph et al.: Phys. Rev. Lett. 80 (1998) 3018 and this proceedings

40. N. Carjan et al.: "The Nucleus: New Physics for the New Millennium" eds. Smit et al., Kluwer Academic/Plenum Publishers, New York (2000) 115

41. D.Strottman et al.: Phys. Scripta, T88 (2000) 148 